\title{
Urological Disorders
}

\section{Siniša Franjić*}

Faculty of Law, International University of Brcko District, Bosnia and Herzegovina

Research Article

Volume 4 Issue 2

Received Date: April 09, 2019

Published Date: May 03, 2019

DOI: 10.23880 /oajun-16000163 Brcko District, Brcko, Bosnia and Herzegovina, Europe, Tel: +387-49-49-04-60; Email: sinisa.franjic@gmail.com

\section{Abstract}

Urology is a branch of clinical medicine that deals with etiology, pathogenesis and diagnosis of urinary tract disorders in men and women, and reproductive organs in men. Physician specialists in this area are called urologists and he has been trained to diagnose and treat urogenital disorders. Urogenital tract disorders under Urology include urinary tract infections, kidney and urinary bladder with various bacteria, as well as men's prostate inflammation, kidney function disorders such as urinary traps, birth anomaly, kidney stone, benign and malignant kidney tumors. This paper was created according to the rules of the desk research methodology in which the author elaborated the available medical literature in the field of urology. The result of the study is the treatment that the doctor must take when a malignant patient comes to him.

Keywords: Urology; Medicine; Health; Injury

Abbreviations: CEPOD: Confidential Enquiry Into Perioperative Deaths, GUM: Genitourinary Medicine, STIs: Sexually Transmitted Infection, PN: Partner Notification, RCT: Randomized Controlled Trial, IVP: Intravenous Pyelogram.

\section{Introduction}

The organs of the male and female urinary tracts are mostly contained within the abdomen [1]. They are divided into: those of the upper urinary tract, the kidneys and their drainage tubes (the ureters); and those of the lower urinary tract, the bladder and its drainage tube (the urethra). The organs of the male genital tract are mainly external. They consist of the testes and their drainage system - the epididymes and vas deferens as contained in the scrotum - and the prostate gland, seminal vesicles and ejaculatory ducts, as draining into the posterior portion of the urethra immediately below the bladder outlet.
The organs of the female genital tract consist of the ovaries, fallopian tubes and uterus. The uterus is divided into fundus, body and cervix. The cervix is inserted into the vault of the vagina which itself opens into the introitus of the female external genitalia. The organs of the male and female urinary tract are anatomically the same until they reach their final drainage portion, the urethra. Here, the urinary tract becomes closely associated with the genital tract such that, in the male, the urethra serves additional genital functions.

Urological disorders account for about one third of all surgical admissions to hospital [2]. Urological pathology is also a common reason for patients to present in primary care. Although few urological conditions are immediately life threatening, many may have a profound effect on the patient's quality of life.

As with all other medical and surgical specialties, subspecialisation has occurred within urological practice. 
Evidence in the confidential enquiry into perioperative deaths (CEPOD) highlighted that transurethral prostatectomy, the operation performed most often in urological departments, is associated with a significantly lower mortality when performed by surgeons who undertake more than 50 such procedures a year. Most urologists will undertake core urology and will subspecialise in one or two of the component parts of urology. One common theme is that urological surgery requires specialised urological nursing to be effective.

\section{Discussion}

\section{Urological Health}

The investigation and treatment of patients with urological disorders tends to be minimally invasive. The urological equivalent of diagnostic laparotomy seldom, if ever is needed. Most of even the most major urological surgical procedures may be performed laparoscopically. This will become the standard in the next few years. The pharmaceutical industry is anticipating an increase in the proportion of medically treated urological disorders: from $5 \%$ to $15 \%$ over the next 10 years. With an ageing population and increased expectation for quality of life, the demand for medical and surgical urological care is likely to increase. Manpower predictions for training purposes will become increasingly complex.

Shared care between urologists and primary care doctors is common and effective. Integration with other healthcare professionals-such as district nurses, physiotherapists, radiographers, and urology nurse practitioners based in hospitals-also has a pivotal role. One recent example of close collaboration is the setting up of multidisciplinary tumour group meetings to manage patients with urological cancers. The provision of urological health is likely to shift in the next few years. The role of the "independent sector" in the provision of non-oncological urology is unclear, and the latter may become the "Cinderella" of the specialty.

\section{Genitourinary Medicine}

The ultimate goal of Genitourinary Medicine (GUM) is to reduce the incidence of STIs (sexually transmitted infection) within the community [3]. This is achievable by providing accessible and non-judgmental services that provide free and immediate diagnosis and treatment to those who think they may have, or have been at risk of an STI. Epidemiological control of infection remains essential to the sexual welfare of the community. However, clinics must reconcile confidentiality with a need for care and hospital services to be aware of serious chronic health problems in the individual. Health advising with good community links is important for ensuring that PN (partner notification) issues are dealt with efficiently and sensitively, reducing the continued transmission of STIs.

Comprehensive national surveillance programmes are essential when formulating strategies for national screening and treatment policies. Surveillance data informs clinical practice, allows the planning and allocation of resources and helps identify at-risk populations.

The management of acute genitourinary tract infections has dramatically changed because of advances in diagnostic testing and antibiotic availability [4]. In the past, the ability to recognize and treat lower urinary tract and genital infections required diagnostic acumen, mostly based on physical findings. Now, sophisticated molecular and radiological tests facilitate the recognition of acute prostatitis, urethritis, and epididymo-orchitis. These entities usually involve common pathogenic organisms and high-risk patient populations. However, each disease entity has a distinct pathology, necessitating individual evaluation and management.

\section{RCT}

Evidence-based clinical practice has been defined as the "conscientious, explicit and judicious use of current best evidence in making decisions about the care of individual patients" [5]. Clinical decision making should combine patient preferences and values with the best available evidence when making treatment choices for individual patients. Inherent in this philosophy of practice is that a hierarchy of evidence exists; certain study types provide higher quality evidence than others. A central tenet of evidence-based practice is that a hierarchy of evidence exists. Among individual studies, the randomized controlled trial (RCT) provides the highest level of evidence, although ideally a meta-analysis of several RCTs will provide better estimates of treatment effects than a single RCT. Below the RCT in the hierarchy of evidence come cohort studies, which follow groups of patients through time. The key difference between cohort studies and RCTs is that patients are not randomly allocated to treatments in a cohort study. Cohort studies may be prospective i.e., patients are allocated into cohorts prior to the occurrence of the primary outcomes.

The hierarchy of evidence exists because individual study designs are inherently prone to bias, that is, systematic deviation from the truth. As opposed to random error, bias has a magnitude and a specific direction. Bias may serve to over- or underestimate treatment effects, and therefore lead to erroneous 
conclusions about the value of therapeutic interventions. RCTs sit at the top of the hierarchy of evidence because well-designed and executed RCTs contain the strongest methodological safeguards against bias. Study designs further down the hierarchy of evidence are subject to increasing potential for bias, and therefore constitute lower levels of evidence.

Randomized controlled trials are unique in the hierarchy of evidence, as participants in the trial are not selected for specific interventions but instead are allocated randomly to a specific therapy or control. With appropriate methodological safeguards, RCTs have the potential to provide the highest level of evidence for questions of therapy.

\section{Urological Injuries}

When the urologist is called to the operating room for consultation on a possible urologic injury, he should do several things before scrubbing up [6]. First, he should read the chart for any relevant history that may affect the patient's urologic status. Second, he should ask if any preoperative radiologic studies, such as an intravenous pyelogram (IVP), are available for review.

After scrubbing up, the urologist should assess the situation. If exposure is poor, there should be no reluctance about repositioning retractors or extending the surgical incision, if necessary. In addition, if hemostasis is inadequate, the urologist should ensure a relatively dry surgical field before proceeding. The ureter should be identified and traced out above and below the area of concern. The urologist should be aware that, if a ureter has been injured, the contralateral ureter or bladder might also have been injured; depending on the circumstances, these structures should also be examined intraoperatively. Indigo carmine or methylene blue may be given intravenously to aid in checking the integrity of the ureters; these dyes may be instilled via a urethral catheter to help identify or confirm bladder injuries.

\section{Clinical Practice Guidelines}

Clinical practice guidelines are an important tool for achieving optimal patient care. Since the emergence of the concept of evidence-based medicine [7], healthcare providers have sought ways to synthesize evidence into formats and products that are both valid and readily implemented into routine practice. Rigorously developed, high-quality (i.e. trusted) practice guidelines have the potential to promote the use of effective clinical services, decrease undesirable practice variation, reduce the use of services that are of minimal or questionable value, increase the use of effective but underused services, and target services to populations most likely to benefit. Guidelines do not supplant the clinical judgment of physicians, rather they provide guidance based on the best available evidence.

Clinical practice guidelines can influence a large number of healthcare providers and patients so their quality is critically important. The quality of practice guidelines refers to the confidence that the potential biases of guidelines development have been addressed adequately, recommendations are both internally and externally valid, and the guidelines are feasible for practice. High-quality guidelines are valid (unbiased), reproducible, clinically applicable to the populations of interest, flexible, clearly presented, developed through a multidisciplinary process, reviewed on a regular schedule, and well documented.

The quality of practice guidelines depends on the quality and rigor of each of the steps performed in formulating the guidelines: crafting of the key clinical questions to be addressed in the guidelines, identification of studies for inclusion, assessment of the quality of individual studies, synthesis of the body of evidence and assessment of its quality, and the formulation of recommendations or practice guidelines.

Clinical practice guidelines and the recommendations contained therein are distinct from, but should usually be based on, systematic reviews of the clinical questions addressed in the guidelines. High-quality systematic reviews provide an exhaustive, unbiased synthesis of the available evidence. Guidelines present recommendations about which approaches should or should not be taken in specific clinical circumstances based on the evidence identified in the systematic review. There are thus two separate junctures where guideline developers must provide a transparent, reliable, and valid assessment: quality or grade of the body of evidence identified in the systematic review process which reflects the level of confidence that if a recommendation is followed, the anticipated outcome will occur; and second, assessment of the strength of recommendations, which takes into account the balance of benefits and harms and the importance of adhering to the recommendations.

There are many types of questions that a urologist needs to ask (or be asked in turn by a patient or relative) [8]. These may include questions concerning etiology, diagnosis, prognosis, harm, effectiveness, and qualitative outcomes. Different questions require different study designs. To find out what living with a condition (e.g., advanced prostate cancer) is like, a qualitative study that 
explores the patient experiences is required. In contrast, a qualitative study relying only on the subjective experiences of individuals could be unhelpful when trying to establish whether an intervention or treatment works. The best design for effectiveness is the randomized controlled trial (RCT). A hierarchy of evidence exists, published by the Oxford Centre for Evidence Based Medicine, by which different methods of collecting evidence are graded as to their relative levels of validity. The design of a study (such as a case report for an individual patient or a double-blind randomized control trial) and the end points measured (such as survival or quality of life) affect the strength of the evidence. A crosssectional survey is a useful design to determine the frequency of a particular condition. However, when determining an accurate prognosis for someone diagnosed with, say, lower urinary tract symptoms, a cross-sectional survey (that observes people who have the disease and describes their condition) can give a biased result. A design more suited for a prognosis in question is an inception cohort-a study that follows up a recently diagnosed patient and records what happens to them over an extended period of time.

\section{Responsibility}

Litigation after any operation more often than not arises because something unexpected has happened [9]. It may be a known complication of the operation that the surgeon may not find surprising, or simply a poor outcome, but whatever it is, it happens entirely unanticipated by the patient. The patient believes, or the relatives believe, that something has gone wrong which ought not to have gone wrong. They then interpret this as having been due to an error by the surgeon, and go on to make the assumption that such an error on his part must have involved negligence. Litigation may then follow.

The judgment as to whether an act or omission is negligent is a subtle one, and is not exercised harshly against the practitioner who has done his best and been unlucky. It has to be related to a bed-rock of basic standards of knowledge below which one cannot go. Negligence in medicine or surgery has been defined straightforwardly by the courts as the making of an error (by act or omission) of which no ordinary doctor/surgeon, professing the relevant skill, would have been guilty, if acting with reasonable care.

We know the law exists, but we do not often think about it in our everyday lives even though it is continuously in operation, for example, when goods are bought and sold, when people get married or companies are formed [10]. The law lays down rules in respect of all of these matters. When things are done in the usual way there is little reason to worry. In the normal course of events people only begin to consider the law when some uncertainty or difficulty arises. When a person looks into their situation after a difficulty has arisen they may find it is probably too late. The law, by its nature, applies retrospectively. It is usually when something has gone wrong that the law steps in.

Everyday decisions are made about patient care, planning and treatment. Health professionals go about their daily routines and whilst terms like 'accountability' and 'responsibility' form part of their everyday vocabulary they do not sit back and think 'what does it really mean'? But when something goes wrong they become accountable. By its nature accountability applies retrospectively. The discharge of accountability rests with the courts. The health professional cannot go the courts beforehand and say 'if I do something in this way, will I be in trouble'? In reality they go about the daily tasks weighing up risks and making decisions and hope that when they do so, the courts, the employer and the professional body will uphold what they do. Being forewarned is being forearmed and understanding the law and accountability will help the health professional make the right decisions.

The health professional must know the area of law that affects them. Every health professional must know that ignorance of the law is no defence. Very broadly speaking, the law is a set of rules.

\section{Conclusion}

Pain from kidney or urethra is usually strictly limited to the loins and the spine, and it can spread to the same orifice region, upper thighs, testis, or labia. The kidney stones typically cause colic, often spread out, whose intensity less waves in infection. Acute urinary retention caused by changes distal from the bladder causes intense suprapubic pain, while chronic retention is often asymptomatic. Diaphuria is a symptom of irritation of the bladder or urethra. The pain from the prostate is reported by an uncomfortably limited discomfort and feeling of fullness of the perineal, rectal or suprapubic regions. Symptoms of urinary bladder obstruction include delayed urination, strain, urine drop and drip at the end of the urination.

\section{References}

1. Smith P (2001) Urology. Cavendish Publishing Limited, London, UK, pp: 11. 


\section{Open Access Journal of Urology \& Nephrology}

2. Whitfield HN (2006) Introduction to urology. In: Dawson C, Whitfield HN (Eds.), ABC of Urology, 2nd (Edn), Blackwell Publishing Ltd, Malden, USA, pp: 911.

3. Pattman R, Snow M, Handy P, Sankar KN, Elawad B (2005) (Eds.), Oxford Handbook of Genitourinary Medicine, HIV and Aids, Oxford University Press, Oxford, UK, pp: 4.

4. Tran KB, Wessells H (2005) Genital and Infectious Emergencies - Prostatitis, Urethritis, and EpididymoOrchitis. In: Wessells H, McAninch JW (Eds.), Urological Emergencies - A Practical Guide, Humana Press Inc, Totowa, USA, pp: 135.

5. Scales ChD, Penson DF (2010) Clinical trials in urology. In: Dahm Ph, Dmochowski RR (Eds.), Evidence-Based Urology, Blackwell Publishing Ltd, John Wiley \& Sons Ltd, Chichester, UK, pp: 12.

6. Loughlin KR (2006) Recognition and management of urologic complications of gynecologic surgery. In: Cardozo L, Staskin D (Eds.), Textbook of Female
Urology and Urogynecology, 2nd (Edn.), Informa Healthcare, Abingdon, UK, pp: 1369.

7. Norris SL (2010) Evidence-based guidelines in urology. In: Dahm Ph, Dmochowski RR (Eds.), Evidence-Based Urology, Blackwell Publishing Ltd, John Wiley \& Sons Ltd, Chichester, UK, pp: 38-39.

8. O'Flynn KJ (2010) Evidence-Based Medicine. In: Mundy AR, Fitzpatrick JM, Neal DE, George NJR (Eds.), The Scientific Basis of Urology, $3^{\text {rd }}$ (Edn.), Informa Healthcare, London, UK, pp: 560.

9. Gotley RG, Reynard JM, Badenoch J (2007) Urology And The Law - Lessons From Litigation. Informa Healthcare, CRC Press, Taylor \& Francis Group, Boca Raton, USA, pp: 9.

10. Lynch J (2009) Clinical Responsibility, CRC Press, Taylor \& Francis Group, Boca Raton, USA, pp: 23. 